\title{
SIMULATION-BASED EVALUATION OF SUSCEPTIBILITY DISTORTION CORRECTION METHODS IN DIFFUSION MRI FOR CONNECTIVITY ANALYSIS
}

\author{
Oscar Esteban ${ }^{1,2, *}$, Alessandro Daducci ${ }^{2}$,Emmanuel Caruyer ${ }^{3}$, Kieran O'Brien ${ }^{4}$, \\ María J. Ledesma-Carbayo ${ }^{1}$, Meritxell Bach-Cuadra ${ }^{5,2}$, and Andrés Santos ${ }^{1}$ \\ ${ }^{1}$ Biomedical Image Technologies (BIT), ETSI Telecom., U. Politécnica de Madrid and CIBER-BBN, Spain. \\ ${ }^{2}$ Signal Processing Laboratory (LTS5), École Polytechnique Fédérale de Lausanne (EPFL), Lausanne, Switzerland. \\ ${ }^{3}$ Section of Biomedical Image Analysis, Dpt. of Radiology, University of Pennsylvania, Philadelphia, PA, USA. \\ ${ }^{4}$ Centre d'Imagerie Biomédicale (CIBM), and Dpt. of Radiology, University of Geneva, Geneva, Switzerland. \\ ${ }^{5}$ Radiology Department, CIBM, Lausanne University Hospital Center (CHUV) and Lausanne University (UNIL), Switzerland. \\ ${ }^{*}$ Corresponding author (email: oscar.esteban@upm.es)
}

\begin{abstract}
Connectivity analysis on diffusion MRI data of the wholebrain suffers from distortions caused by the standard echoplanar imaging acquisition strategies. These images show characteristic geometrical deformations and signal destruction that are an important drawback limiting the success of tractography algorithms.

Several retrospective correction techniques are readily available. In this work, we use a digital phantom designed for the evaluation of connectivity pipelines. We subject the phantom to a "theoretically correct" and plausible deformation that resembles the artifact under investigation. We correct data back, with three standard methodologies (namely fieldmap-based, reversed encoding-based, and registrationbased). Finally, we rank the methods based on their geometrical accuracy, the dropout compensation, and their impact on the resulting connectivity matrices.
\end{abstract}

Index Terms - susceptibility artifacts, diffusion MRI, tractography, connectivity.

\section{INTRODUCTION}

In-vivo whole-brain connectivity analysis has been a research topic of high interest for the last $5 \mathrm{yr}$. Diffusion MRI (dMRI) can be used to probe the orientation of fiber bundles within the brain, and it is generally acquired with an echo-planar imaging (EPI) sequence. After signal reconstruction, tractography algorithms draw a map of the sampled structures. These maps can represent the actual trajectories of fiber bundles (deterministic tractography) or pixel-wise probability of connection to a certain origin (probabilistic tractography). Finally, the information about these connections is collected into a network matrix that is subjected to the "connectome analysis".

Among all the difficulties that such a complex workflow raises [1], we will address here the susceptibility-derived artifacts, for which EPI schemes are especially sensitive. Magnetic susceptibility disturbs the magnetic field close to tissue interfaces. This inhomogeneity of the field translates into a highly distorted anatomy and a significant signal destruction in certain regions of the brain (e.g. the orbitofrontal lobe, for the proximity of the air surrounding sinuses). This artifact has been well described, generally within the context of functional MRI, which also uses EPI.
Various approaches have been proposed to correct for this distortion. Fieldmap-based (FMB) methods [2] rely on one extra acquisition (field mapping), that probes the inhomogeneity of the B0 field. A second theory-based breed of methodologies acquire a map of the point-spread function of the EPI readouts to correct for the artifact [3]. Another approach referred to as reversed encoding-based (REB), acquires an extra EPI volume in the orthogonal or reversed phase encoding that can be combined to remove the geometric distortions $[4,5]$. The last set of methodologies uses an extra T2-weighted image as anatomical reference to seek for the deformation map through nonlinear registration $[6,7]$. These T2w registration-based (T2B) methods usually map the $\mathrm{T} 2 \mathrm{w}$ image to the baseline volume or $b 0$ of dMRI, as the latter exhibits a contrast very similar to the anatomical $\mathrm{T} 2 \mathrm{w}$. More recent works report extensions or combinations of existing techniques [8-11].

Even though the aforementioned techniques for distortion correction have been studied $[12,13]$, the lack of a goldstandard limits benchmarking strategies. Recently, Irfanoglu et al. [14] raised the question of distortion-derived impact on tractography. In this work, we propose an evaluation framework using a digital phantom designed for connectivity assessment. This framework enabled us to compare several correction techniques and characterize their geometrical accuracy and the dropout compensation. Finally, we report their impact on subsequent tractography and connectivity.

\section{METHODS}

\subsection{Digital Phantom}

Based on the fiber geometries of the digital phantom created for the HARDI Reconstruction Challenge held in ISBI 2013 (San Francisco, US), we simulated high resolution $(0.5 \mathrm{~mm}$ isotropic) $\mathrm{T} 1$ weighted $(\mathrm{TE} / \mathrm{TR}=10 / 1500 \mathrm{~ms})$ and $\mathrm{T} 2 \mathrm{w}(\mathrm{TE} / \mathrm{TR}=90 / 5000 \mathrm{~ms})$ images, as well as two dMRI images (1.0mm isotropic, $b=1200,1 b 0$ image) with 32 and 64 evenly-distributed directions intended for diffusion tensor imaging (DTI) and high angular resolution diffusion imaging (HARDI) reconstructions, respectively. Diffusion is modeled by a restricted and a hindered compartment, similar to [15]. The phantom includes white matter (WM) fiber bundles, grey matter (GM) and cerebrospinal fluid (CSF). 
Physical properties (T1/T2 times in ms) used in simulation are $(832 \pm 10 / 79 \pm 0.6)$ for $\mathrm{WM},(1331 \pm 13 / 110 \pm 2.0)$ for $\mathrm{GM}$ and $(3500 \pm 100 / 250 \pm 10)$ for CSF. T1w, T2w and diffusion weighted images (DWIs) were added normally distributed noise up to a signal-to-noise ratio (SNR) of $30 \mathrm{~dB}$.

\subsection{Theory-based synthetic distortion}

FMB methodologies use a map of the field in the scanner. More precisely, the phase difference between two subsequent samplings of the field map. With that information, it is possible to compute the theoretical displacement that each voxel undergoes, the so-called voxel shift map (VSM). The most prominent feature of this VSM is that all the shifts have the same orientation (parallel to the phase-encoding direction of the EPI) and their magnitude and direction depend on the EPI gradient increments (or blips) and the actual phase difference at the voxel.

In order to create a realistic distortion, we generated a synthetic and noise-free phase-difference map consistent with the phantom, using the tools distributed with the FSL package (www.fmrib.ox.ac.uk/fsl) and standard parameters $(\Delta \mathrm{TE}=2.46 \mathrm{~ms}$. for the field mapping and effective dwell time of $0.77 \mathrm{~ms}$ for the DWI). We defined two regions of smooth dephasing and computed the corresponding VSM. Amplitude of the dephasing maps can be modulated, enabling us to evaluate the magnitude of distortion. We generated several VSMs with increasing maximum shifts (from 3.80 to $7.60 \mathrm{~mm}$ ), covering the typical magnitudes of distortion observed in real datasets.

From these synthetic VSMs, we generated the corresponding distorted DWIs, in two opposed phase-gradient encoding directions. The second simulated "acquisition" of the same phantom was necessary for evaluating REB methods.

In summary, we generated a full gold-standard containing realistic T1w and T2w at high resolution, DWIs acquired in two different phase encoding directions, and a ground-truth DWI data, which is not available for real datasets (Figure 1).

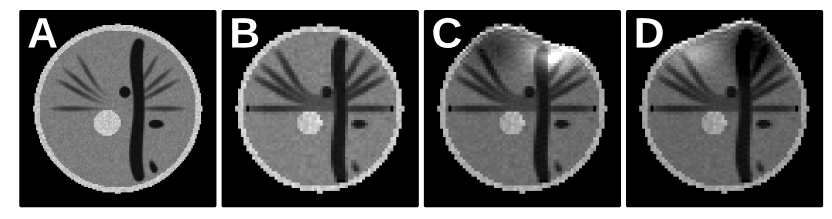

Fig. 1. Ground-truth digital phantom. A) T2w; B) undistorted $b 0$ volume; $\mathrm{C}, \mathrm{D})$ distorted $b 0$ volumes with opposed phase encoding directions, maximum displacement of $3.80 \mathrm{~mm}$.

\subsection{Correction methods}

Three out of four methods presented in section 1 were tested on the evaluation framework. FMB correction is the same as the one used for generating the distortion, working on the opposite direction. Normally distributed noise was added to the synthetic field map ( $\mathrm{SNR}=20 \mathrm{~dB})$ before correction, for a more realistic evaluation. REB correction is implemented in $F S L$ (topup), and it demands for the $b 0$ of the reverse-encoding simulation. In this second case, the VSM is inferred from the differences between the two corresponding $b 0$. Finally, we included T2B methods with ANTs (stnava.github.io/ANTs), fine-tuned for $b 0$-to-T2w registration. To this end, we used a multi-resolution scheme with 3 levels of subsampling and smoothing, mutual information metric, and the symmetric diffeomorphic transform model. Several configurations of kernel widths for the regularization smoothers were tested, and finally selected $0.5 / 1.0$ voxels (gradient/deformation fields, respectively) for its best result. Additionally, undistorted images are corrected for dropout using the determinant of the Jacobian of the deformation field.

\subsection{Evaluation}

The original phantom, one distorted version, and the corrected instances are then connected to a DWI reconstruction and tractography pipeline. Additionally, the original tissue probability maps are also distorted and corrected to provide tractography with the required WM masks. These maps are also used in a final assessment module.

The framework supports two different methods for DWI reconstruction and deterministic tractography: 1) Diffusion Toolkit (trackvis.org/dtk) for DTI, is configured with 10 random seeds per voxel by default; and 2) MRTrix (www.brain.org.au/software/mrtrix) for HARDI, with default parameters set to use constrained spherical deconvolution, maximum harmonic order of 6, and 150000 desired tracks. For both options, the seeding regions can be set to use either the distorted-corrected WM mask, or the regions used to generate the ground-truth. This second seeding strategy mimics the usual procedure on real data, where regions are typically mapped from the anatomically correct T1w.

The evaluation framework is completed by automated assessment modules. We evaluated three characteristics of the correction methods. Firstly, we assessed the geometrical correctness reporting overlap indices of three tissue probability maps (namely CSF, WM, and GM), weighting the average by tissue volumes. Secondly, to evaluate the quality of the actual signal dropout correction, we studied the similarity volume by volume computing the $\ell_{1}$-norm correlation index. We report this score on the $b 0$ and the average of the remaining DWI volumes. Thirdly, we studied the impact on the connectivity matrices reporting the number of false positives (nonexistent connections in the gold-standard) and false negatives (or missed connections).

\section{RESULTS}

All the modules described in the previous section were conveniently integrated in workflows using nipype (nipy. sourceforge.net/nipype). The choice of this tool grants the reproducibility of the experiments, and the evaluation workflow is publicly released. The results of this study are summarized in Figure 2 and Table 1. The remaining of this section provides extended descriptions and interpretation of these findings. 


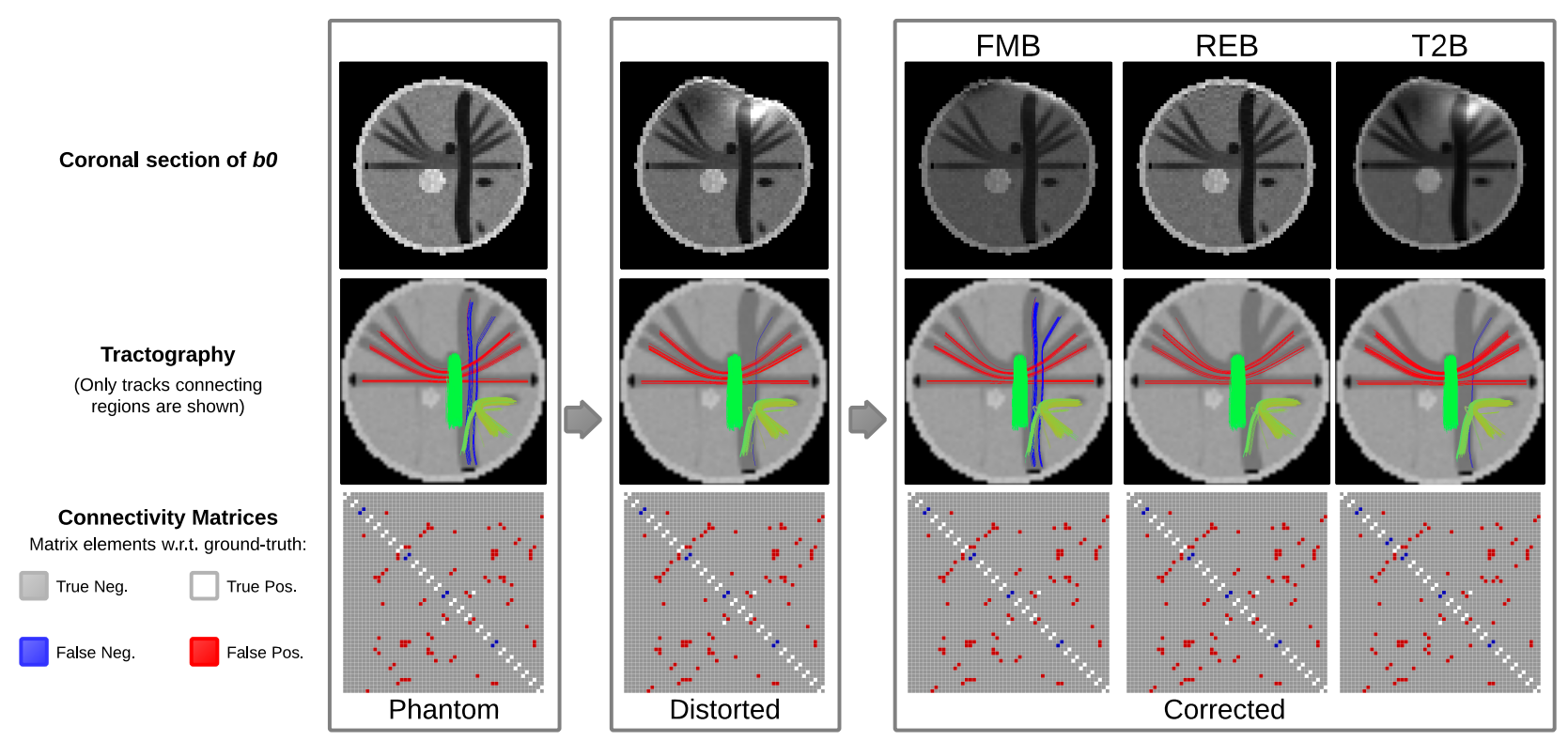

Fig. 2. Visual assessment of the correction methods.

Table 1. Accuracy results

\begin{tabular}{c|cccc|cc}
\hline & \multicolumn{3}{||}{ Overlap (Jaccard Index, \%) } & \multicolumn{2}{c}{ Signal Correlation (\%) } \\
\hline & Av. & CSF & WM & GM & b0 & DWIs \\
\hline FMB & 93.00 & 88.57 & 96.74 & 94.02 & 80.05 & $96.26 \pm .06$ \\
\hline REB & 96.64 & 94.31 & 98.26 & 96.75 & 91.00 & $97.65 \pm .03$ \\
\hline T2B & 79.19 & 66.31 & 89.85 & 82.14 & 64.58 & $90.10 \pm .13$ \\
\hline
\end{tabular}

Table 2. Tractography and connectivity results.

\begin{tabular}{l||cccc}
\hline & \# tracks & length $(\mathrm{mm})$ & FP & FN \\
\hline Original & 735 & $40.87 \pm 13.55$ & 40 & 4 \\
\hline Distorted & 878 & $40.54 \pm 13.73$ & 42 & 4 \\
\hline FMB & 743 & $40.04 \pm 13.60$ & 43 & 4 \\
\hline REB & 830 & $39.87 \pm 13.93$ & 44 & 4 \\
\hline T2B & 825 & $41.44 \pm 12.85$ & 40 & 5 \\
\hline
\end{tabular}

\subsection{Geometrical correctness and signal recovery}

A summary of quantitative results, computed for the DTI dataset with max. shift $3.80 \mathrm{~mm}$, is provided in Table 1 . The best scores were obtained with the REB method, followed by FMB. The clear difference of accuracy between REB and FMB with respect to T2B infers the latter may not be an appropriate method for susceptibility correction. The described performance was constant for FMB and REB along the different magnitudes of distortion evaluated (max. shift 3.80-7.60 mm).

A second workflow investigated the similarity of the recovered signal with respect to the original (undistorted). In this second study, REB performed better than the other two methods, as reported in Table 1. REB scored a 91.0\% similarity index for the $b 0$ volume and an average $97.65 \%$ for the remaining DWIs. Again, the second qualified was FMB, which achieved very close results for the DWIs (96.26\%) but not as good for the $b 0$ volume. Visual inspection of the recovered data confirms the presented quantitative results (Figure 2).

\subsection{Impact on tractography and connectivity}

Connectivity matrices derived from the DTI dataset were completely hindered by the high complexity of the fibers contained in the phantom. Tractography discovered successfully only 4 out of 27 existing connections from the original (undistorted) data, with more than 45 false connections. With the HARDI dataset we found 23/27 connections, but we still observed 40 false positives. Therefore, the experiments using the DTI phantom were discarded. The results presented in this subsection only refer to the HARDI dataset.

Using the strongest distortion (max. shift $7.60 \mathrm{~mm}$ ), the number of detected connections remained the same (23/27), slightly increasing the number of false positives to 42 . The immediate conclusion is that, with highly complex anatomies (crossing, fanning, etc.) and limited number of ground-truth connections, connectivity matrices are more sensitive to reconstruction and tractography than to distortions.

For the sake of completeness, Table 2 reports the characteristic features of the tractography results and connectivity matrices, for the fieldmap that caused a max. shift of $3.80 \mathrm{~mm}$. A very similar outcome was obtained for $7.60 \mathrm{~mm}$. These results, along with visual inspection (Figure 2), might point to FMB as the best correction method.

\subsection{Discussion}

Even though all the surveyed methods produced visually sound results, our study suggested that REB is the most accurate method in terms of geometrical agreement and signal dropout recovery. The T2B method did not achieve the necessary high-standards to recommend its use. Nonetheless, we understand that specific methods with anisotropic 
regularization that completely restrict deformations to the phase-encoding direction would perform significantly better than the standard method presented. Geometrical correctness of DWI data is fundamental in connectivity analysis to spatially locate the regions which will define the nodes of the final connectivity matrix.

Regarding tractography, this study revealed that signal reconstruction and tractography algorithms masked the impact of susceptibility distortion on the final connectivity matrices. Due to this effect, experiments performed on the DTI phantom were discarded for comparison. With the HARDI dataset, the extracted connectivity matrix slightly changed with distortion. Quantitative differences reported in Table 2 could be more related to the smoothing derived from interpolation implemented by each method. Visual results might suggest that FMB achieved better results.

Although we found connectivity matrices rather invariant with respect to distortion, they are suspected to be significantly impacted by the susceptibility artifact on real data [14]. This hypothesis points to the need of more appropriate phantoms with denser connectivity matrices. State of the art phantoms for tractography usually present a discrete set of simulated fiber bundles that translate in very sparse fiber-endings regions and connectivity matrix. In the real case, the surface limiting the tracks is densely covered by the regions mapped from the anatomical dataset, what leads to larger sensitivity with respect to deformations.

A possible limitation of this work is that FMB is used for both synthesis and correction of the distortion. However, practical reasons (i.e. noise, signal dropout) impede perfect geometrical correction with FMB. Moreover, REB performed more accurately than FMB.

Future extensions of this work will include a refined digital phantom. Additional lines for this work will evaluate different realizations of the synthetic phase-difference map, to better characterize the phenomenon. Also, the framework can be enhanced for studying the impact of other artifacts as subject's motion or eddy currents-derived distortions.

\section{CONCLUSION}

This paper proposes an evaluation framework to comprehensively analyze the impact of susceptibility induced distortion on connectivity analyses from dMRI data. Inaccuracy on tractography produces errors and a increased variability in the extracted connectivity matrices of the whole-brain. This effect is suspected to influence those regions with larger distortions. However, this study found out that the connectivity matrix is more largely affected by the tractography method. We publicly release the framework and also contribute with the evaluation of the most widely-used and readily-available correction methodologies. The reverse encoding-based method achieved the best results correcting for susceptibility-induced artifacts. We also reported visual evaluation of tractography and quantitative results for the extracted connectivity matrices.

\section{REFERENCES}

[1] D. K. Jones et al., "Twenty-five pitfalls in the analysis of diffusion MRI data," NMR in Biomed., 23(7):803-820,
2010.

[2] P. Jezzard et al., "Correction for geometric distortion in echo planar images from B0 field variations," Magn. Reson. Med., 34(1):65-73, 1995.

[3] M. D. Robson, et al., "Measurement of the point spread function in MRI using constant time imaging," Magn. Reson. Med., 38(5):733-740, 1997.

[4] D. Cordes, et al., "Geometric distortion correction in EPI using two images with orthogonal phase-encoding directions," in ISMRM, vol. 8, p. 1712, 2000.

[5] J. Y. Chiou et al., "A simple method to correct offresonance related distortion in echo planar imaging," in ISMRM, vol. 8, p. 1711, 2000.

[6] J. Kybic, et al., "Unwarping of unidirectionally distorted EPI images," IEEE Trans. Med. Imag., 19(2):80-93, 2000.

[7] C. Studholme, et al., "Accurate alignment of functional EPI data to anatomical MRI using a physics-based distortion model," IEEE Trans. Med. Imag., 19(11):11151127, 2000.

[8] J. L. Andersson, et al., "How to correct susceptibility distortions in spin-echo echo-planar images: application to diffusion tensor imaging," NeuroImage, 20(2):870888, 2003.

[9] M. Zaitsev, et al., "Point spread function mapping with parallel imaging techniques and high acceleration factors: Fast, robust, and flexible method for echo-planar imaging distortion correction," Magn. Reson. Med., 52(5):1156-1166, 2004.

[10] D. Holland, et al., "Efficient correction of inhomogeneous static magnetic field-induced distortion in echo planar imaging," NeuroImage, 50(1):175, 2010.

[11] J. L. R. Andersson, et al., "A comprehensive gaussian process framework for correcting distortions and movements in diffusion images," in ISMRM, vol. 20, p. 2426, 2012.

[12] H. Zeng et al., "Image distortion correction in EPI: comparison of field mapping with point spread function mapping," Magn. Reson. Med., 48(1):137-146, 2002.

[13] M. Wu, et al., "Comparison of EPI distortion correction methods in diffusion tensor MRI using a novel framework," in MICCAI, vol. 5242, pp. 321-329, 2008.

[14] M. O. Irfanoglu, et al., "Effects of image distortions originating from susceptibility variations and concomitant fields on diffusion MRI tractography results," $\mathrm{Neu}$ rolmage, 61(1):275-288, 2012.

[15] Y. Assaf et al., "Composite hindered and restricted model of diffusion (CHARMED) MR imaging of the human brain," NeuroImage, 27(1):48-58, 2005. 